\title{
Intra-articular glenohumeral injections of HYADD®4-G for the treatment of painful shoulder osteoarthritis: a prospective multicenter, open-label trial
}

\author{
GIUSEPPE PORCELLINI ${ }^{1}$, GIOVANNI MEROLLA ${ }^{1,2}$, NICOLA GIORDAN ${ }^{3}$, PAOLO PALADINI ${ }^{1}$, \\ ANDREA BURINI ${ }^{3}$, EUGENIO CESARI ${ }^{4}$, ALESSANDRO CASTAGNA $^{4}$ \\ ${ }^{1}$ Unit of Shoulder and Elbow Surgery, D. Cervesi Hospital, Cattolica (RN), AUSL della Romagna Ambito Rimini, Italy \\ 2 "Marco Simoncelli" Biomechanics Laboratory D. Cervesi Hospital, Cattolica (RN), AUSL della Romagna Ambito Rimini, Italy \\ ${ }^{3}$ Research and Development Department - Fidia Farmaceutici SpA, Abano Terme (PD), Italy \\ ${ }^{4}$ Unit of Orthopaedics, Istituto Clinico Humanitas, Rozzano (MI), Italy
}

\begin{abstract}
Purpose: numerous experimental and clinical studies in osteoarthritis (OA) have demonstrated that intraarticular (IA) administration of hyaluronic acid can improve the altered rheological properties of the synovial fluid and exert protective and reparative effects on the joint structure. The objective of this study was to evaluate the safety and performance of HYADD ${ }^{8} 4-G\left(\right.$ Hymovis $\left.{ }^{\circledR}\right)$ in patients with glenohumeral joint $\mathrm{OA}$.

Methods: forty-one patients with shoulder pain and limited shoulder function resulting from concentric glenohumeral joint $\mathrm{OA}$ were enrolled in a multicenter clinical trial. Patients received two HYADD ${ }^{\circledR} 4-G$ injections administered one week apart. The main outcome measure was improvement in shoulder pain on movement at six months as assessed through a $100-\mathrm{mm}$ visual analog scale (VAS), range of motion (ROM) values, and Constant-Murley Shoulder Outcome Score (CS).

Results: two IA injections of HYADD ${ }^{\circledR} 4-\mathrm{G}$ (Hymovis $\left.{ }^{\circledR}\right)$ significantly decreased pain and improved shoulder function for up to six months from the first injection. The VAS score decreased (from $66.1 \mathrm{~mm}$ to $37.7 \mathrm{~mm}$ at six months) and improvements were recorded in the total CS and in the ROM values ( rotation
\end{abstract}

\section{Corresponding Author:}

Giovanni Merolla, MD

Unit of Shoulder and Elbow Surgery, "D. Cervesi"

Hospital

Via L. V. Beethoven 5, 47841 Cattolica (RN), Italy

E-mail: giovannimerolla@hotmail.com decreased from a mean value of $54.2^{\circ}$ at baseline to $63.2^{\circ}$ at six months and internal rotation from a mean value of $44.0^{\circ}$ at baseline to $45.7^{\circ}$ at 26 weeks). No serious adverse events occurred.

Conclusions: the study results demonstrated that two IA injections of HYADD ${ }^{\circledR} 4-G$ (Hymovis ${ }^{\circledR}$ ) may be a safe and effective treatment option for shoulder pain associated with glenohumeral OA and that the effects of the injections are still present for up to six months after the treatment.

Level of evidence: Level IV, therapeutic case series.

Keywords: shoulder, osteoarthritis, intra-articular injections, hyaluronan, painful shoulder.

\section{Introduction}

Osteoarthritis $(\mathrm{OA})$ is a highly debilitating degenerative joint disease. As life expectancy increases, $\mathrm{OA}$ is becoming a major cause of limitation of physical activity, which, in turn, can have psychological effects. Loss or reduction of shoulder functionality can lead to the development of anxious-depressive syndromes as well as affecting the ability to work (1-3).

Current conservative treatment for shoulder pain due to OA includes rest and physical therapy $(4,5)$, oral non-steroidal anti-inflammatory drugs (NSAIDs) (47 ), intra-articular (IA) corticosteroids $(4,5,7)$ and IA sodium hyaluronates $(8,9)$.

Surgical treatment options for shoulder OA, especially the prosthetic option, are still being improved. In patients presenting early pathology onset, contraindi- 
cations to surgical treatments, or initial osteoarthritic degeneration, it may be appropriate to choose a conservative approach such as IA hyaluronan injections. Viscosupplementation is a non-surgical treatment capable of improving painful symptoms and leading to recovery, even partial, of glenohumeral joint mobility. A large number of experimental and clinical studies in OA have demonstrated that IA administration of hyaluronic acid (HA) may improve the altered rheological properties of the synovial fluid and exert protective and reparative effects on the joint structure (10-22). Hyaluronan is a naturally occurring linear polysaccharide composed of repeating disaccharide units of $\mathrm{D}$ glucuronic acid and $\mathrm{N}$-acetyl-D-glucosamine. HA is synthesized by type-B synoviocytes in the synovial lining and released into the joint cavity, where it plays a functional role, providing lubrication and cushioning during joint movement (10). The course of OA joint diseases is characterized by the appearance of typical degenerative changes in the cartilage, synovial membrane, synovial fluid and subchondral bone.

Several HA-based products are now available on the market and there is substantial clinical evidence demonstrating their efficacy and safety in knee and hip OA treatment (23-27). However, only a few studies, using a limited number of different products, have evaluated the efficacy of HA in shoulder OA treatment.

The objective of this study was to evaluate the feasibility, safety and efficacy of a novel HA chemical derivative (HYADD®4-G; Fidia Farmaceutici SpA, Italy) in patients with chronic shoulder pain and limitation of motion due to glenohumeral joint OA. This multicenter clinical trial, although open and uncontrolled, set out to establish whether the effectiveness and safety demonstrated with HYADD4-G during a pivotal study in knee OA could be replicated in OA of the shoulder (28).

\section{Methods}

\footnotetext{
Study design

This prospective, multicenter, open-label study was designed to determine whether two ultrasound-guided IA injections of HYADD ${ }^{\circledR} 4-G$ in patients with $\mathrm{OA}$ of the shoulder provide significant reduction in pain during activity at up to six months from baseline as measured using a $100-\mathrm{mm}$ visual analog scale (VAS) for pain.
}

Local ethics committee approval and written informed consent were obtained prior to the patients' enrolment and the study was conducted in accordance with the Helsinki Declaration of 1975 and subsequent revisions.

\section{Participants}

Forty-one patients with bilateral chronic shoulder pain due to OA entered the study, but only one shoulder (the target one) was considered for the purposes of this study. The criteria for inclusion in the study were: 45 years of age or older; radiographic confirmation of $\mathrm{OA}$; chronic shoulder pain for at least six months but less than five years; pain perceived with a frequency of at least $50 \%$ of the days in the month preceding the inclusion evaluation; moderate to severe pain as indicated by a rating of 40 to $80 \mathrm{~mm}$ on the VAS; limitation of active range of motion ( $\mathrm{ROM})$ in at least one direction (i.e. abduction with scapula fixed $\leq 80^{\circ}$; internal rotation in abduction $\leq 55^{\circ}$; external rotation in abduction $\leq 80^{\circ}$ ). Musculoskeletal system-related reasons for exclusion from the study included: presence of adhesive capsulitis; evidence of rotator cuff lesions; presence of primary extra-articular shoulder syndromes; detection of clinically significant shoulder deformities; acute fractures; chondrocalcinosis; cervical spine disorders; IA corticosteroid injections to the target joint in the previous three months, or in any other joint in the month prior to the inclusion evaluation; IA hyaluronan injections within the previous month; severe OA of the glenohumeral joint.

Pregnancy, malignant disease, any situation that might affect the subsequent clinical evaluations (such as treatment with local radiotherapy for breast cancer; prior history of any malignancy of the skin), and laboratory abnormalities indicating thyroid insufficiency were also reasons for exclusion. All analgesics, bisphosphonates and oral pharmaceutical products containing glucosamine, chondroitin sulphate or diacerein had to be discontinued two months prior to the baseline evaluation. A radiograph of the shoulder performed either at screening or within the previous three months was used to confirm the diagnosis of $\mathrm{OA}$ and to rule out the presence of any exclusion criteria such as fractures, severe loss of bone density or severe deformity. The integrity and function of the rotator cuff was verified by clinical assessment and X-ray examination, but when required, an MRI scan was also performed. 


\section{Interventions}

HYADD ${ }^{\circledR} 4-G\left(\right.$ Hymovis $^{\circledR}$; Fidia Farmaceutici SpA, Italy) is supplied as an aqueous formulation of

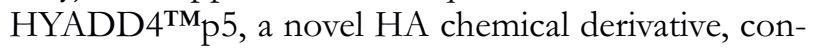
taining a low number of $\mathrm{C} 16$ side-chains per polysaccharide backbone that provides injectable hydrogels stabilized by side-chain hydrophobic interactions. The HA employed as the initial material in the chemical

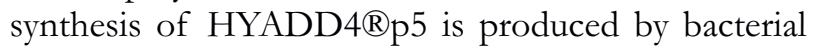
fermentation of a non-pathogenic strain of Streptococcus equi and is characterized by a molecular weight of about 500 to $730 \mathrm{kDa}$ (32). Articular injections were administered using a posterior shoulder approach under ultrasound guidance. The patient was seated with the arm in adduction, the shoulder was disinfected with an iodine solution and a 22-gauge, 1.25-inch needle attached to the syringe was introduced $2 \mathrm{~cm}$ inferior and $1 \mathrm{~cm}$ medial to the postero-inferior acromial angle, and directed $20^{\circ}$ medially and caudally, until the articular surface was reached.

\section{Outcome measures}

Patients were evaluated on inclusion (V0), at baseline (V1) and at one week (V2), 13 weeks (V3), 17 weeks (V4) and 26 weeks (V5) after the initial treatment.

At the inclusion visit (V0), the following information was recorded: demographics, baseline characteristics, medical history, complete OA history, bilateral shoulder assessment of the ROM, target shoulder radiography if not performed within the three months prior to inclusion, OA severity grade, review of the use of concomitant treatments for the painful shoulder or any other clinical condition(s), complete physical examination including blood pressure and heart rate. The patient was also provided with a diary card to report his/her daily intake of rescue medication. The patient was instructed not to take any rescue medication within the 24 hours prior to a clinical assessment.

Each patient received two IA injections of HYADD ${ }^{8} 4-G$ (prefilled syringe containing $3 \mathrm{ml}$ of sodium hyaluronate in phosphate buffered saline) administered a week apart; one injection at baseline (V1) and the other at week 1 (V2).

Clinical assessments at baseline and at all subsequent visits included a target shoulder examination, during which any swelling, tenderness, presence of effusion redness/inflammation or pain during activity were recorded, and assessment of the ROM of the target shoulder.

Follow-up efficacy assessments at V3, V4 and V5 included measurement of target shoulder pain during activity and at night using the $100-\mathrm{mm}$ VAS, administration of the EQ-5D questionnaire and ConstantMurley Shoulder Outcome Score (CS).

Safety evaluations were performed recording any adverse events (AEs) experienced by the patient in the minutes subsequent to the IA administration and during the study, as well as any changes in concomitant medications and use of rescue medications.

The primary study objective was to determine whether ultrasound-guided IA injections of Hymovis ${ }^{\circledR}$ provide significant reduction in shoulder pain during activity at up to six months from baseline as measured on a $100-\mathrm{mm}$ VAS for pain. The secondary end point was the reduction in pain perceived during the night at up to six months from baseline as assessed by VAS.

Additional end points included improvement of shoulder functionality as assessed by ROM and CS up to six months from baseline. The local and general safety of the treatment was also assessed.

\section{Data analysis}

Efficacy variables were analyzed using descriptive statistics of raw values and variation from baseline at each visit. Comparison against baseline at all time-points was assessed by appropriate tests for repeated measures at an a level of 0.05 or adjusted for multiple comparisons if needed (the Bonferroni method was adopted).

Safety was assessed through descriptive statistics of vital signs and summary of AEs.

\section{Results}

All 41 enrolled patients suffered from chronic shoulder pain with limitation of motion due to glenohumeral joint OA. Thirty patients (73\%) were male, and all the patients were Caucasian. Their mean age was 65 years and their mean body mass index was 24.7 $\mathrm{kg} / \mathrm{m}^{2}$. Eighteen patients (54\%) reported concomitant diseases at trial entry, the most common being hypertension ( 8 patients; $20 \%$ ) and diabetes ( 3 patients; $8 \%)$. At inclusion, only 5 patients (12\%) suffered from bilateral glenohumeral joint OA; limitation of shoulder motion was shown by the following mean values: 
external rotation $52.4^{\circ}$, internal rotation $44.3^{\circ}$, abduction $96.5^{\circ}$, full neutral abduction $118.6^{\circ}$, forward flexion $125.8^{\circ}$ and posterior reach: $5.4 \mathrm{~cm} .61 \%$ of the patients had grade $2 \mathrm{OA}$ and $37 \%$ had grade 3 . At the baseline visit, the patients had a mean VAS pain score during activity of $65.7 \mathrm{~mm}$ and a mean VAS pain score at night of $49.4 \mathrm{~mm}$.

Pain during activity measured by VAS was the primary study outcome. At baseline, patients reported a mean score of $65.7 \mathrm{~mm}$, which decreased at each evaluation to reach a mean of $42.5 \mathrm{~mm}$ at 26 weeks. At 13 weeks (V3), the patients showed a significant decrease in pain perceived during activity compared to baseline; this significance was maintained at week 17 (V4) and week 26 (V5) ( $<<0.05)$. Compared to the baseline value, the VAS pain score showed mean decreases of $17.4 \mathrm{~mm}$ at V3, $21.2 \mathrm{~mm}$ at $\mathrm{V} 4$, and $23.2 \mathrm{~mm}$ at V5.

The improvement in pain during activity was even more evident in the per protocol population where the mean value decreased from $66.1 \mathrm{~mm}$ at baseline to $37.7 \mathrm{~mm}$ at 26 weeks. The VAS pain scores at 13,17 , and 26 weeks (respectively V3, V4 and V5) were significantly different from the value observed at baseline $(\mathrm{p}<0.05)$. Compared to the baseline value, the VAS pain score showed mean decreases of $20.6 \mathrm{~mm}$ at V3, $22.3 \mathrm{~mm}$ at $\mathrm{V} 4$, and $28.3 \mathrm{~mm}$ at V5.

Pain during the night also improved, showing mean decreases compared to baseline of $17.9 \mathrm{~mm}$ at V3, $17.3 \mathrm{~mm}$ at V4, and $18.0 \mathrm{~mm}$ at V5 (p<0.05) (Fig. 1). As regards ROM, which was measured at each study time point, all movements improved from baseline to V5: external rotation improved from a mean value of $54.2^{\circ}$ at baseline to a mean value of $63.2^{\circ}$ at V5; internal rotation from a mean value of $44.0^{\circ}$ at baseline to a mean value of $45.7^{\circ}$ at $\mathrm{V} 5$; abduction with scapula fixed from a mean value of $94.8^{\circ}$ at baseline to a mean value of $102.7^{\circ}$ at V5; full neutral abduction from a mean value of $117.6^{\circ}$ at baseline to a mean value of $133.9^{\circ}$ at V5; forward flexion from a mean value of $127.0^{\circ}$ at baseline to a mean value of $148.7^{\circ}$ at V5; posterior reach from a mean value of $5.5 \mathrm{~cm}$ at baseline to a mean value of $6.9 \mathrm{~cm}$ at V5 (Fig. 2).

An improvement in the EQ-5D weighted index, against baseline, was observed at each study time point with mean increases of 2, 4 and 3\% recorded at V3, V4 and V5.

In addition, the CS increased at each study time point, from a mean value of 43.0 at baseline to a mean value of 54.5 at V5 (Fig. 3). Overall the treatment was well tolerated. Nineteen AEs were reported during the study in 14 patients but none of them was considered related to the study product. The most common AEs were flu (mild or moderate and reported by six patients) and mild headache (experienced by 3

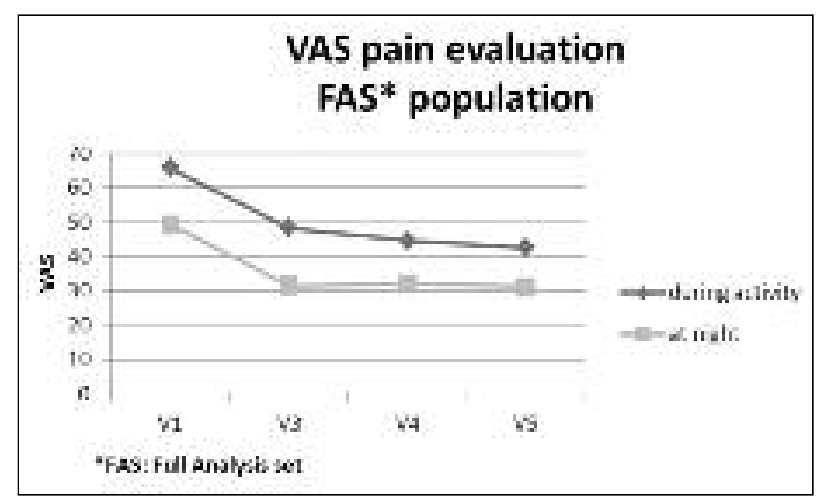

Fig. 1. VAS Pain evaluation during activity and night.
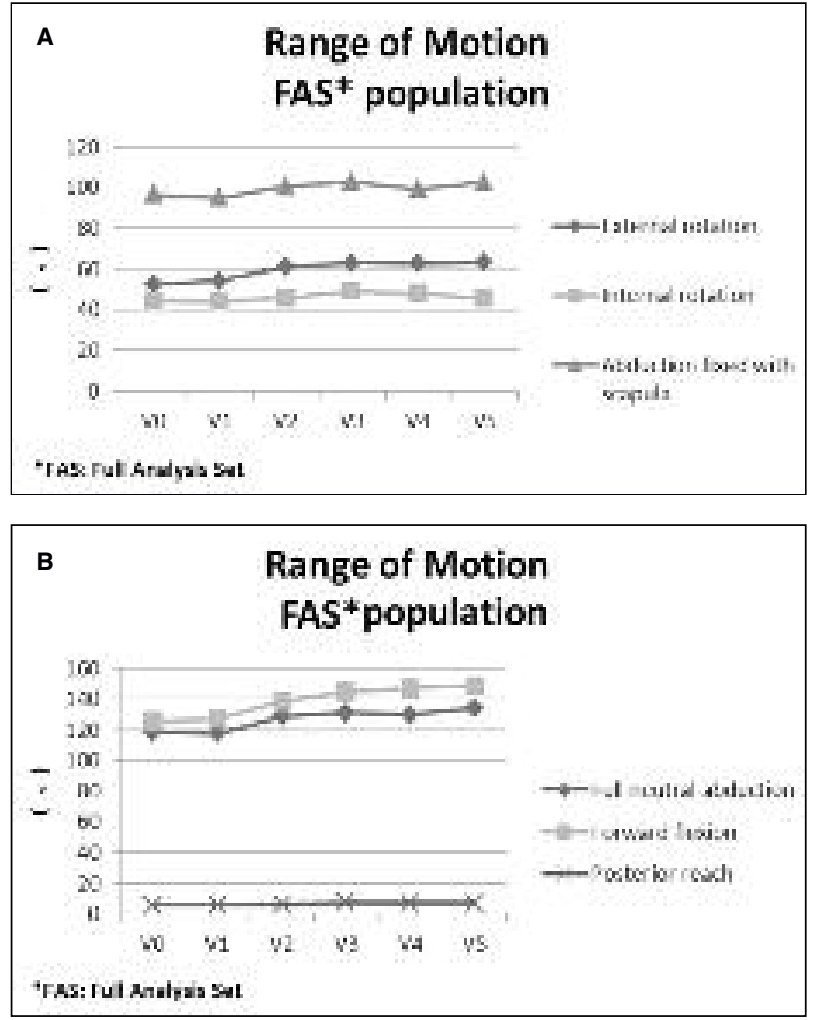

Fig. 2. Range of motion evaluation. A: External rotation, internal rotation and abduction with scapula fixed. B: Full neutral abduction, forward flexion, and backward extension 


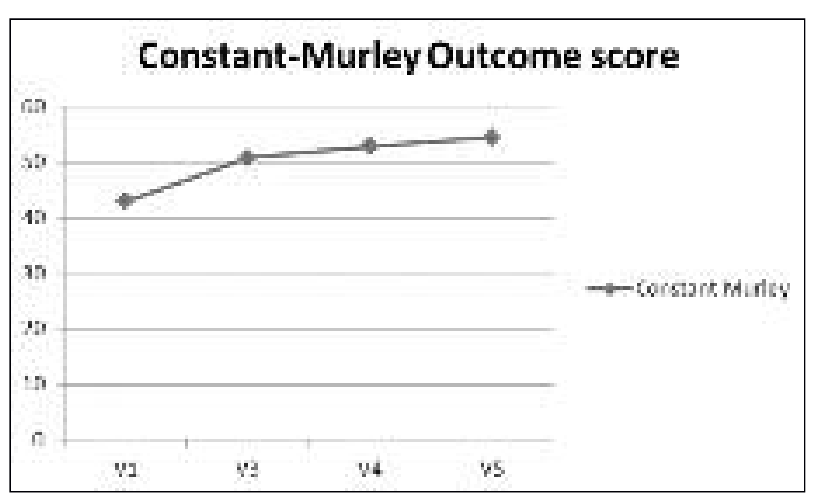

Fig. 3. Constant-Murley Shoulder Outcome Score.

patients). During the course of the study just two serious AEs occurred, neither of which was related to HYADD ${ }^{\circledR 4-G . ~}$

\section{Discussion}

Glenohumeral joint OA is a gradual and progressive disease and, at present, no known intervention ultimately alters the progression of the disease. Non-surgical treatments are therefore directed towards reducing symptoms and improving functionality.

The literature contains descriptions of the use of HA derivatives with different molecular weights in the treatment of shoulder $\mathrm{OA}$; these studies reported good improvements in patient satisfaction and quality of life (29-31). In all of these studies, the Authors assessed the effectiveness of HA viscosupplementation in relation to the grade of $\mathrm{OA}$ and to the molecular weight of the HA derivative. The preparation used in this study (HYADD®4-G) is a novel HA in which $2-3 \%$ of the disaccharide repeating units have been modified by introducing hexadecylamine as a side-chain on the CDI-activated carboxyl group of the glucuronic unit; this modification results in a partially hydrophobized HA that provides hydrogels (32). HYADD ${ }^{\circledR} 4-G$ has been shown to be associated with a significantly better amelioration of IL- $1 \beta$-induced gene expression of key matrix degrading enzymes (MMP1, MMP13, ADAMTS5), and inflammatory mediators (IL-6, PTGS2) by human OA chondrocytes and synovial fibroblasts (33).

In this study we investigated the impact of
HYADD ${ }^{\circledR} 4-G$ on shoulder pain and functionality in patients with chronic shoulder pain and limitation of motion due to glenohumeral OA.

The results from this open-label study on 41 patients showed that two IA injections of Hymovis ${ }^{\circledR}$ administered one week apart significantly decrease pain and improve shoulder functionality for up to six months from the first injection. Pain, total CS and ROM all showed significant improvements at three months.

In consideration of the increase in shoulder function observed in the passive and active ROM, the reduction in perceived pain, and the improvement in global function, it is clear that the results of this study indicate an overall improvement in glenohumeral functionality.

Our results are partially consistent with the available literature (34). A recent systematic review on IA injection therapy for patients with glenohumeral OA showed good efficacy of $\mathrm{HA}$ at follow-up during the first six months; this review also highlighted the low recommendation for the use of corticosteroid injections in patients with glenohumeral OA. The Authors agreed that there is a need, in future research based on powered randomized trials, to compare the efficacy of HA, steroids and placebo solutions in shoulder OA (34).

Given its ease of use, the absence of adverse events related to HYADD4-G IA treatment and the results obtained, it is possible to assume that two IA injections of HYADD4-G $\left(\right.$ Hymovis $^{\circledR}$ ) may be a safe and effective treatment option for shoulder pain due to concentric glenohumeral OA. Because of the slight recurrence of pain around the 6th month, we might consider suggesting a second cycle of IA injections 67 months after the first.

This study has two main limitations: first, the absence of a control group; second, the small sample size. Prospective randomized controlled trials are necessary to provide exhaustive evidence of the long-term efficacy of HA-derived products in glenohumeral OA treatment in a larger population.

\section{Acknowledgements}

Data collection and analysis, the reporting and writing of this study were supported by a grant from Fidia Farmaceutici SpA. No equipment or other supplies were provided by Fidia Farmaceutici SpA. The Authors declare no conflict of interest with respect to the contents of this article. 


\section{References}

1. Memel DS, Kirwan JR, Sharp DJ, et al. General practitioners miss disability and anxiety as well as depression in their patients with osteoarthritis. Br J Gen Pract. 2000;50:645-648.

2. Matsen FA 3rd, Ziegler DW, DeBartolo SE. Patient self-assessment of health status and function in glenohumeral degenerative joint disease. J Shoulder Elbow Surg. 1995;4:345-351.

3. Nakagawa Y, Hyakuna K, Otani S, et al. Epidemiology study of glenohumeral osteoarthritis with plain radiography. J Shoulder Elbow Surg. 1999;8:580-584.

4. van der Windt DA, Koes BW, de Jong BA, et al. Shoulder disorders in general practice: incidence, patient characteristics, and management. Ann Rheum Dis. 1995;54:959-964.

5. Lyons PM, Orwin JF. Rotator cuff tendinopathy and subacromial impingement syndrome. Med Sci Sports Exerc. 1998;30(4 Suppl):S12-17.

6. Friis J, Jarner D, Toft B, et al. Comparison of two ibuprofen formulations in the treatment of shoulder tendonitis. Clin Rheumatol. 1992;11:105-108.

7. Adebajo AO, Nash P, Hazleman BL. A prospective double blind dummy placebo controlled study comparing triamcinolone hexacetonide injection with oral diclofenac $50 \mathrm{mg}$ TDS in patients with rotator cuff tendinitis. J Rheumatol. 1990;17:12071210.

8. Itokazu M, Matsunaga T. Clinical evaluation of high-molecularweight sodium hyaluronate for the treatment of patients with periarthritis of the shoulder. Clin Ther. 1995;17:946-955.

9. Ramonda R, Franceschini M, Leardini G. Treatment of shoulder periarthritis with hyaluronic acid. Riv Ital Biol Med. 1998;1:51 (Suppl 2).

10. Abatangelo G, O'Regan M. Hyaluronan: biological role and function in articular joints. Eur J Rheumatol Inflamm. 1995;15:9 16.

11. Altman RD, Moskowitz R. Intraarticular sodium hyaluronate $($ Hyalgan () in the treatment of patients with osteoarthritis of the knee: A randomized clinical trial. Hyalgan Study Group. J Rheumatol. 1998;25:2203-2212.

12. Carrabba M, Paresce E, Angelini M, et al. The safety and efficacy of different dose schedules of hyaluronic acid in the treatment of painful osteoarthritis of the knee with joint effusion. Eur J Rheumatol Inflamm. 1995;15:25-31.

13. Dougados M, Nguyen M, Listrat V, et al. High molecular weight sodium hyaluronate (Hyalectin $\left.{ }^{\circledR}\right)$ in osteoarthritis of the knee: a 1-year placebo-controlled trial. Osteoarthritis Cartilage. 1993; 1:97-103.

14. Huskisson EC, Donnelly S. Hyaluronic acid in the treatment of osteoarthritis of the knee. Rheumatology (Oxford). 1999;38: 602-607.

15. Leardini G, Mattara L, Franceschini M, et al. Intra-articular treatment of knee osteoarthritis: a comparative study between hyaluronic acid and 6 methyl prednisolone acetate. Clin Exp Rheumatol. 1991;9:375-381.

16. Maheu E. Hyaluronan in knee osteoarthritis: A review of clinical trials with Hyalgan. Eur J Rheumatol Inflamm. 1995;15:17-24.

17. Kotz R, Kolarz G. Intra-articular hyaluronic acid: duration of the effect and results of repeated treatment cycles. Am J Orthop (Belle Mead NJ). 1999;28(11 Suppl):5-7.

18. Frizziero L, Govoni E, Bacchini P. Intra-articular hyaluronic acid in the treatment of osteoarthritis of the knee: clinical and morphological study. Clin Exp Rheumatol. 1998;16:441-449.

19. Listrat V, Ayral X, Patarnello F, et al. Arthroscopic evaluation of potential structure modifying activity of hyaluronan (Hyalgan) in osteoarthritis of the knee. Osteoarthritis Cartilage. 1997;5:153-160.
20. Pasquali Ronchetti I, Guerra D, Taparelli F, et al. Morphological analysis of knee synovial membrane biopsies from a randomized controlled clinical study comparing the effects of sodium hyaluronate (Hyalgan ${ }^{\circledR}$ ) and methylprednisolone acetate (Depomedrol $\AA$ ) in osteoarthritis. Rheumatology (Oxford). 2001;40:158-169.

21. Guidolin DD, Pasquali Ronchetti IP, Lini E, et al. Morphological analysis of articular cartilage biopsies from a randomized, clinical study comparing the effects of $500-730 \mathrm{kDa}$ sodium hyaluronate (Hyalgan $\left.{ }^{\circledR}\right)$ and methylprednisolone acetate in primary osteoarthritis of the knee. Osteoarthritis Cartilage. 2001; 9:371-381

22. Jubb RW, Piva S, Beinat L, et al. One-year, randomised, placebo (saline) controlled clinical trial of $500-730 \mathrm{kDa}$ sodium hyaluronate (Hyalgan) on the radiological change in osteoarthritis of the knee. Int J Clin Pract. 2003;57:467-474.

23. Migliore A, Tormenta S, Martin LS, et al. The symptomatic effects of intra-articular administration of Hylan G-F 20 on osteoarthritis of the hip: clinical data of 6 months follow-up. Clin Rheumatol. 2006;25:389-393.

24. Stitik TP, Blacksin MF, Stiskal DM, et al. Efficacy and safety of hyaluronan treatment in combination therapy with home exercise for knee osteoarthritis pain. Arch Phys Med Rehabil. 2007; 88:135-141.

25. Phiphobmongkol V, Sudhasaneya V. The effectiveness and safety of intra-articular injection of sodium hyaluronate (500-730 $\mathrm{kDa}$ ) in the treatment of patients with painful knee osteoarthritis. J Med Assoc Thai. 2009;92:1287-1294.

26. Foti C, Cisari C, Carda S, et al. A prospective observational study of the clinical efficacy and safety of intra-articular sodium hyaluronate in synovial joints with osteoarthritis. Eur J Phys Rehabil Med. 2009;47:407-415.

27. Vetro A, Iovane A, Di Gesu M, et al. Pain relief and functional recovery over a six-month period after intra-articular injection with sodium hyaluronate (Mw 1500 - $2000 \mathrm{Kda}$ ) in osteoarthritis of the knee. Eur J of Musculoskel Dis. 2014;3:25-33.

28. Pavelka K, Niethard FU, Giordan N. A multicentre, international, double blind, randomized, placebo-controlled study to assess the efficacy and safety of two different regimens of HYADD4$\mathrm{G}$ in knee osteoarthritis [Abstract 326]. Osteoarthritis Cartilage. 2010;18 (Suppl 2): S144.

29. Merolla G, Sperling JW, Paladini P, et al. Efficacy of Hylan G-F 20 versus 6-methylprednisolone acetate in painful shoulder osteoarthritis: a retrospective controlled trial. Musculoskeletal Surg. 2011; 95:215-224.

30. Blaine T, Moskowitz R, Udell J, et al. Treatment of persistent shoulder pain with sodium hyaluronate: a randomized, control trial. A multicenter study. J Bone Joint Surg Am. 2008;90:970979.

31. Silverstein E, Leger R, Shea KP. The use of intra-articular Hylan G-F 20 in the treatment of symptomatic osteoarthritis of the shoulder: a preliminary study. Am J Sports Med. 2007;35:979985.

32. Finelli I, Chiessi E, Galesso D, et al. A new viscosupplement based on partially hydrophobic hyaluronic acid: a comparative study. Biorheology. 2011;48:263-275.

33. Smith MM, Russell AK, Schiavinato A, et al. A hexadecylamide derivative of hyaluronan (HYMOVIS $\AA$ ) has superior beneficial effects on human osteoarthritic chondrocytes and synoviocytes than unmodified hyaluronan. J Inflamm (Lond). 2013;10:26.

34. Colen S, Geervliet P, Haverkamp D, et al. Intra-articular infiltration therapy for patients with glenohumeral osteoarthritis: A systematic review of the literature. Int J Shoulder Surg. 2014;8:114-121. 